\title{
Emphysema and lung volume reduction: the role of radiology
}

\author{
Katharina Martini, Thomas Frauenfelder \\ Institute of Diagnostic and Interventional Radiology, University Hospital Zurich, Zurich, Switzerland \\ Contributions: (I) Conception and design: T Frauenfelder; (II) Administrative support: T Frauenfelder (III) Provision of study materials or patients: \\ All authors; (IV) Collection and assembly of data: K Martini; (V) Data analysis and interpretation: All authors; (VI) Manuscript writing: All authors; \\ (VII) Final approval of manuscript: All authors. \\ Correspondence to: Thomas Frauenfelder. Institute of Diagnostic and Interventional Radiology, University Hospital Zurich, Rämistrasse 100 , 8091 \\ Zurich, Switzerland. Email: thomas.frauenfelder@usz.ch.
}

\begin{abstract}
Chronic obstructive pulmonary disease (COPD) is one of the leading causes of death worldwide. One of the main findings is pulmonary emphysema in association with chronic bronchitis. Clinical signs, pulmonary function tests and imaging are the current used methods to diagnose and stage emphysema. Lung volume reduction (LVR) and endoscopic lung volume reduction (ELVR) are the current therapeutic options beside lung transplantation in cases of severe emphysema. Nowadays imaging is one of the key factors for the success of these therapies. Especially quantitative computed tomography (CT) with its increasing possibilities has become a viable tool, providing detailed information about distribution and heterogeneity of emphysema. Other imaging techniques like dual-energy CT (DECT) and functional magnetic resonance (MR) have shown to add functional information. These structural and functional information support thoracic surgeons and interventional pulmonologists in selecting patients and optimizing LVR procedures but also enables the development of new endobronchial therapies. Imaging will further improve the individual outcome by supporting the choice of optimal therapy.
\end{abstract}

Keywords: Lung volume reduction (LVR); endobronchial procedure; computed tomography (CT); preinterventional imaging

Submitted Nov 08, 2017. Accepted for publication May 15, 2018.

doi: $10.21037 /$ jtd.2018.05.117

View this article at: http://dx.doi.org/10.21037/jtd.2018.05.117

\section{Introduction}

Chronic obstructive pulmonary disease (COPD) is defined by the Global Initiative for Chronic Obstructive Lung Disease (GOLD) as a common, preventable and treatable disease that is characterized by persistent respiratory symptoms and airflow limitation that is due to airway and/ or alveolar abnormalities usually caused by significant exposure to noxious particles or gases (1). In order to define disease severity and to guide management of COPD patients' symptom assessment, lung function test (LFT) and frequency of respiratory exacerbations are evaluated. COPD patients are defined by the presence of abnormal pulmonary function (i.e., largely irreversible airflow obstruction), exhibit specific morphologic changes in the central and peripheral airways, lung parenchyma, and pulmonary vasculature $(2,3)$.

Lung changes are evaluated by radiological imaging as computed tomography (CT), lung scintigraphy and magnetic resonance imaging (MRI) (4-9). These techniques have much improved our understanding of the pathophysiology of this disorder, especially magnetic resonance (MR) has risen new insides (10).

New demands on imaging rise since the re-introduction of lung volume reduction (LVR) surgery in the 90th and the innovation in endoscopic lung volume reduction (ELVR) like valves, coils and sealants as well as thermic LVR by vapor as successful therapies for emphysema (11-16). The beneficial effects of LVR by surgical resection of emphysematous lung tissue is caused by its negative impact to respiratory mechanics $(14,17,18)$. Understanding these relations helps in the use of imaging to assess the presence, 

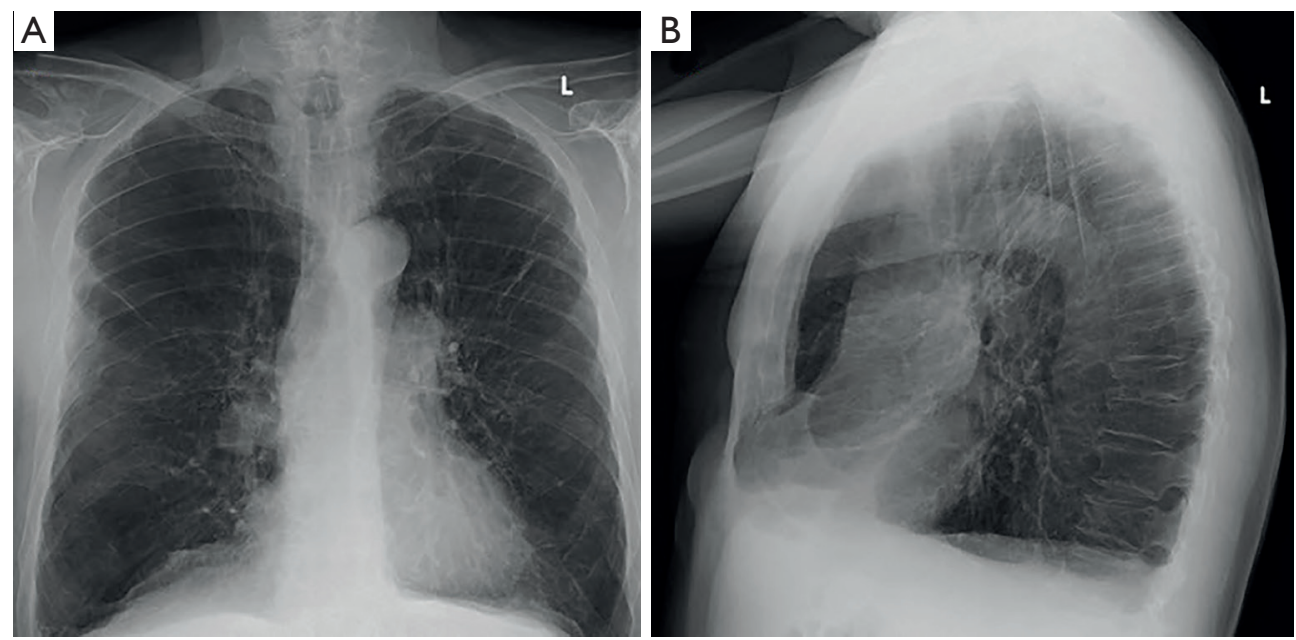

Figure 1 Features of emphysema in conventional radiography. (A) Posteroanterior chest X-ray in a 72-year-old male patient with COPD GOLD III showing hyper-expanded lungs with upper lobe predominant emphysema; (B) lateral projection flattening of the diaphragmatic domes as well as increased anteroposterior diameter and increased retrosternal airspace can be noted. COPD, chronic obstructive pulmonary disease; GOLD, Global Initiative for Chronic Obstructive Lung Disease.

severity, and extent of anatomic alterations and to select appropriate candidates for undergoing LVR $(19,20)$.

Radiographic assessment of patients considering LVR or ELVR has three important functions: (I) establishing the diagnosis of emphysema; (II) to depict extent and distribution of emphysema; (III) to evaluate for other conditions that represent a contraindication to the procedure. But imaging can even more: it enables preoperative planning in terms of quantifying the lung regional amount emphysema and perfusion and simulating the effect of surgical resection $(20,21)$. These information provided by new techniques as for example dual-energy CT (DECT) or functional information from MRI are not yet part of guidelines for COPD diagnosis, prognosis or management, but are discussed for longitudinal studies (22-24). Nevertheless, these information are the decisive element in determining whether a patient is likely to benefit from the LVR procedure. Different studies have shown that the quantification of the extent, the regional distribution of parenchymal destruction, in correlation with perfusion and with functional data are helpful as outcome predictors.

This review provides an overview of current imaging modalities and their role in the evaluation of patients for LVR in terms of diagnostic assessment, preoperative planning and monitoring of outcome. Finally, new tools to enable outcome prediction are addressed.

\section{Role of chest $X$-ray for emphysema and therapy}

The main goal of imaging in line with the evaluation for LVR procedures is to quantify the extent and distribution of emphysema (25).

Findings in chest radiographs that should be evaluated in the initial evaluation of an LVR candidate are additional interstitial pulmonary disease other than emphysema, significant pleural scarring, and pulmonary nodules (Figure 1).

However, the value of posterior anterior (PA) and lateral chest radiograph is limitedly in LVR evaluation: chest radiography has only a sensitivity of $40 \%$ in detecting emphysema (26), and low specificity with high interobserver disagreement hamper a reliable and repeatable quantification of emphysema severity in COPD patients (27). Nevertheless, chest radiography as a fast, widely available, and easy to perform imaging modality at low cost and low radiation dose plays an important role in follow-up of COPD patients. Chest radiography is widely used for postoperative evaluation after LVR or after placement of endobronchial valve, sealant or coil implantation and its early or late complications such as pneumothorax or device displacement. Additionally, chest radiography is essential for pneumothorax management in endobronchial LVR.

For a better depiction of the extent and distribution of emphysema, as well as for a reliable identification of absolute contraindication for LVR procedures additional evaluation with a chest $\mathrm{CT}$ should be performed. 


\section{Role of CT for assessment of emphysema}

\section{Overview}

Chest CT as a fast imaging modality with high temporal resolution and the possibility of $3 \mathrm{D}$ reconstruction and quantification is the imaging modality of choice in the evaluation of COPD. With regard of LVR, CT is used to evaluate the presence of emphysema. It enables the quantification and characterisation of the emphysematous destructed lung (27). These factors are important to give evidence about the effect of LVR for the patient.

Published series, including the National Emphysema Treatment Trial (NETT) (12), showed that patients with upper lobe predominant or heterogeneous emphysema are benefitting the most from LVR. CT is the most important imaging modality to assess heterogeneity and distribution of emphysema in COPD in order to find candidates who will benefit most when undergoing LVR.

CT is also used to identify concomitant conditions/ contraindications to perform LVR such as the presence of malignancy and involvement of airways (i.e., tracheomalacia, mucus plugging, bronchiectasis, lung compression and bronchial wall thickening) (27). In case a suspicious pulmonary nodule is detected on CT, it can be elegantly removed in the setting of LVR. Additionally, expiratory CT is used for the visualization of air trapping but also for the evaluation for bronchial wall collapse during expiration (26).

\section{Quantification}

Emphysema is a destruction of the lung parenchyma distal to the terminal bronchiole, which radiographically are seen as destruction and absence of normal lung parenchyma. Three types of emphysema can be distinguished: (I) panlobular, (II) centrilobular, and (III) septal emphysema and can additionally evaluate the severity and distribution of lung changes (26) (Figure 2). Quantification can be performed by visual scoring, semiquantitative using densitometry or by quantification:

Since visual scoring showed to have a high interobserver variability and is neither sensitive nor precise nowadays mainly semiquantification and quantification is used for the quantification of the amount of emphysema in COPD.

The most widely used semiquantitative method is the Goddard score, whereby the radiologist evaluates the severity of emphysema at three distinct areas in each lung. For each of these six areas, a numerical score of 0 to 4 is assigned, in which 0 represents no emphysema, and 4 more than $76 \%$ of emphysema. In this system, the total score can range from 0 to 24. The heterogeneity of emphysema itself is also assessed at each location. Results from different studies showed a high agreement between readers, if the assessment is performed by experienced radiologists or pulmonologists $(28,29)$.

But other authors report still a high interobserver disagreement of this technique: in the NETT, all observers had to undergo training on a set of CT. Despite the use of experienced and previously trained chest radiologists, there was still significant inter- and intra-observer variability in emphysema quantification (12). To reduce interobserver variability the presentation of the emphysema can be enhanced by simple methods without the use of dedicated software, as for example with sagittal volume rendering based thin slabs, colour coding the severity and distribution of emphysema. This so called "CT densitometry" (Figure 3) has a higher accuracy compared to multiplanar reconstructions (MPRs) (unpublished data).

Nowadays quantitative methods are the standard of reference. Various commercially available software tools can perform automated emphysema quantification. The goal is to identify the lung parenchyma by virtually removing other anatomic structures such as the chest wall and the mediastinum. While CT scanning allows the quantification of tissue density using Hounsfield units (HU), it can be used to assess the density of each voxel within the lung fields. Based on CT characteristics to anatomic characteristics of the lung a threshold is chosen to distinguish normal lung tissue from emphysema $(27,30)$. This threshold ranges normally from -850 to $-950 \mathrm{HU}$. Gevenois et al. $(31,32)$ recommended a cut-off value for emphysematous lung parenchyma on CT scans is $-950 \mathrm{HU}$, which nowadays represents the standard for all different software.

Using a lower threshold than -950 HU would lead to an underestimation of the presence of emphysema, while using a higher threshold than $-950 \mathrm{HU}$ would lead to overestimation of emphysema. Mathematically the amount of emphysema is the percentage of lung tissue below the chosen threshold. Both methods (i.e., the observer and software based approaches) can be used not only the emphysema quantification but also for the classification of the anatomic distribution of emphysema (25).

As already discussed, it is very important for the LVR evaluation to describe the distribution of lung changes as for example in an "upper-lobe predominant" distribution or another distribution (25). In the automated system, the characterization can be done either using a lobe-based 


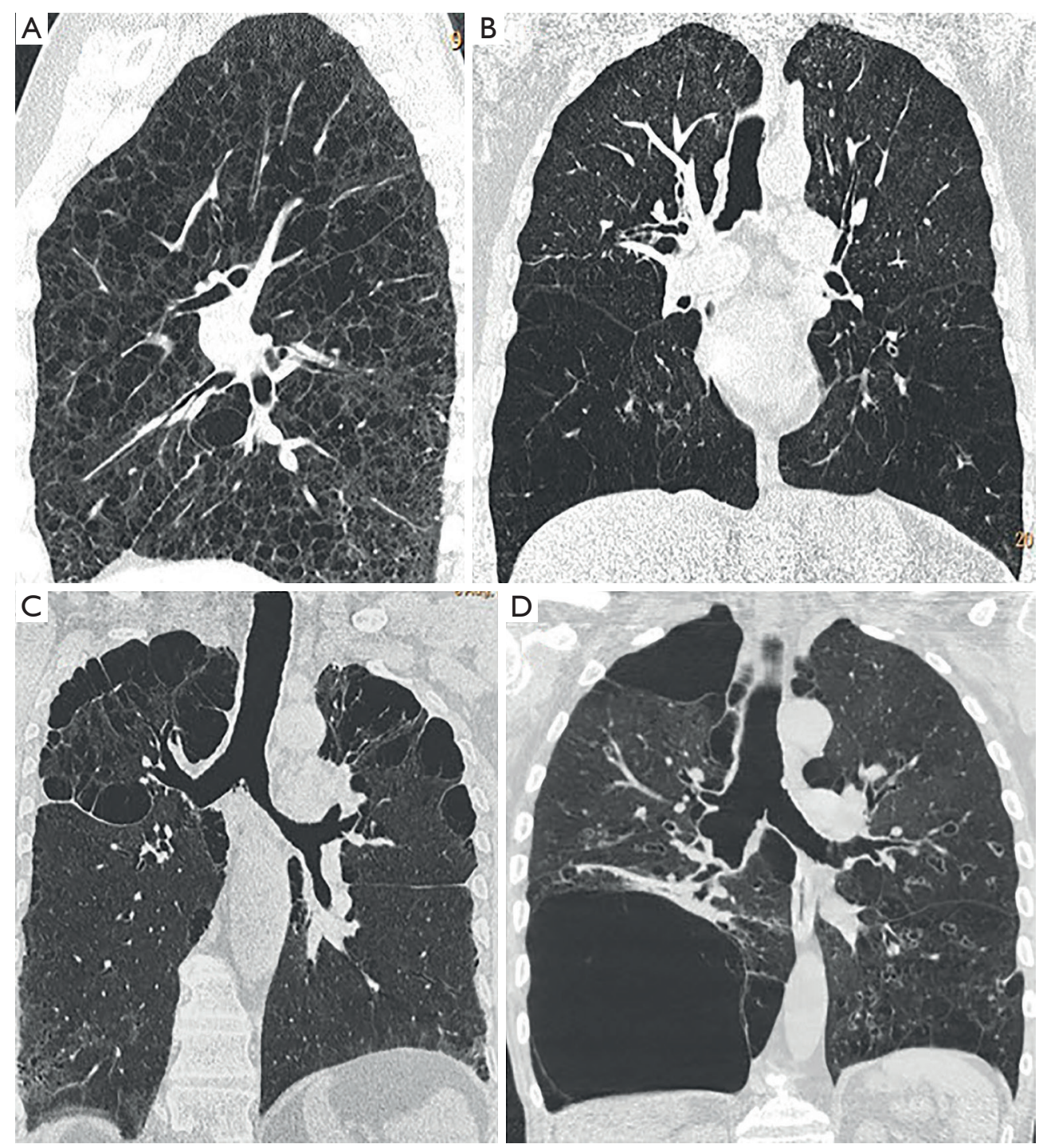

Figure 2 Different emphysema distributions on non-enhanced chest CT. (A) Axial reconstruction in a 63-year-old male patient shows centrilobular emphysema; (B) it shows emphysema in a 58-year-old female patient with panlobular distribution; (C) it shows axial reconstruction in a 67-year-old female patient with paraseptal emphysema; (D) it shows coronal reconstruction in an 82-year-old male patient with bullous emphysema. CT, computed tomography.

quantification or relative by dividing the lung into thirds and used for lung scintigraphy and calculating emphysema heterogeneity by the upper to lower zone ratio of low attenuation areas (LAAs). It has been shown that the preoperative objective LAA ratio correlates with surgical outcomes, as shown by improvement of lung function and maximal exercise (33). Newer techniques allow depicting and quantifying clusters of emphysema, providing inside into the heterogeneity (34). These tools are helpful in characterising the type and distribution of emphysema and allow a primary selection of target areas for LVR or endoscopic interventions. Nevertheless, the validation of the source images remains crucial.

Besides the amount and distribution of emphysema, the integrity of the fissures plays an important role especially for endobronchial valve treatment (19). Incomplete fissures mostly contribute to collateral ventilation. Fissure integrity can be assessed non-invasively with the Chartis Pulmonary Assessment System (35) or non-invasively by using CT. Schuhmann et al. demonstrated that quantitative CT led 

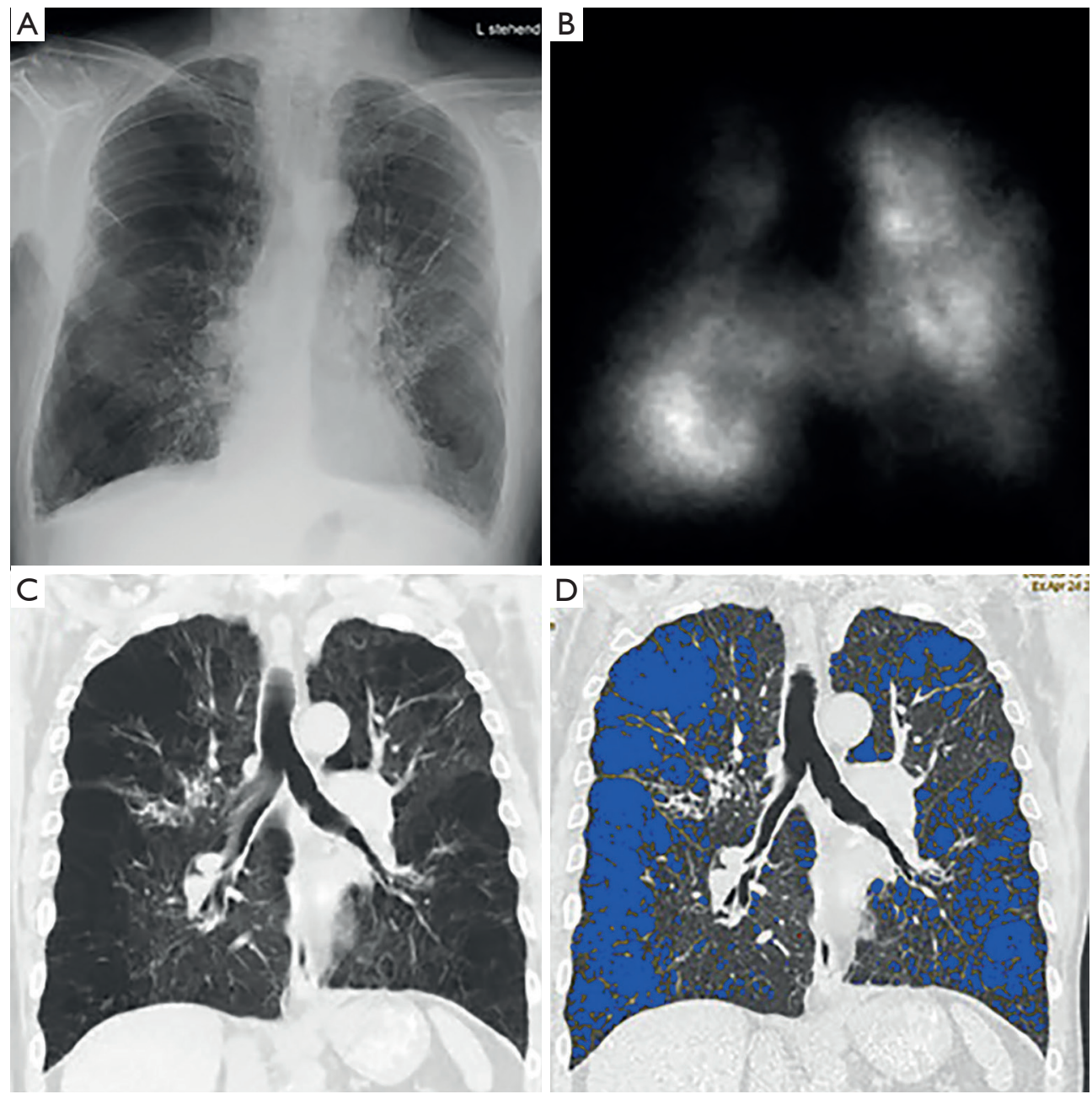

Figure 3 Preoperative imaging before LVR. It shows CT densitometry in a 72-year-old male patient with COPD GOLD III in (A) axial, (B) coronal, and (C) sagittal reconstruction as well as the (D) 3D dataset. The apical predominant distribution of emphysema is highlighted in blue. The higher the score, the higher the presence of emphysema. Lobe area (LAA) and percentage of lobe area to total lung (LAA\%). LVR, lung volume reduction; CT, computed tomography; LAA, low attenuation area; COPD, chronic obstructive pulmonary disease; GOLD, Global Initiative for Chronic Obstructive Lung Disease.

to comparable results to Chartis for classifying LVR and is a valuable method to effectively select suitable patients for valve-based LVR procedures (36). Usually, in cases where fissure integrity is below $95 \%$ by quantitative CT, bronchoscopic measurements using Chartis technology is performed. Complete interlobar fissures were observed only in one-third of patients of a large European study on endobronchial valves (EBV) LVR (13). Although the validation of fissures seems very easy on normal chest CT, it can be very difficult especially in patients with severe emphysema, which is also reflected in a low interobserver agreement (37). In that study, radiologists used MPR imaging in both sagittal and coronal planes, which appeared to be very helpful to assess fissure integrity.

Studies showed that accurate depiction of affected lung parenchyma is crucial for a focalized therapy and to improve treatment outcome: Sciurba et al. reported that patients were HRCT was used to evaluate distribution of emphysema and fissure anatomy to find suitable lung regions for endobronchial valve placements had a better post-interventional outcome that the control-group (15). Different studies show that automated emphysema 

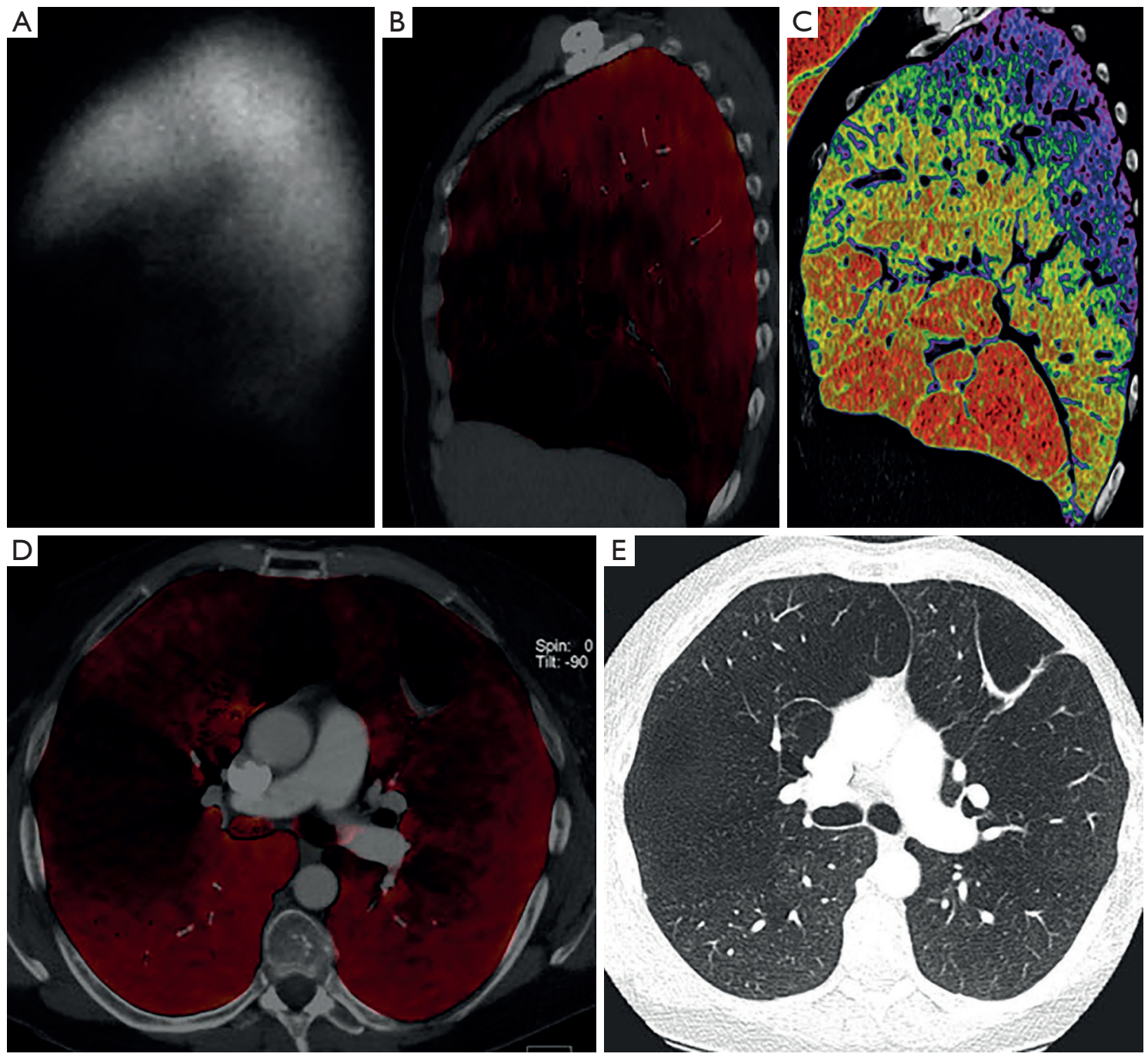

Figure 4 Preoperative imaging before LVR. A 64-year-old male patient with COPD GOLD IV: (A) lower tracer uptake in the lateral perfusion map of lung scintigraphy is nicely correlating with lower attenuation on (B) DECT and (C) CT densitometry on sagittal reconstruction. (D) It shows axial reconstruction of DECT in the same patient and (E) shows the respective axial slice on non-enhanced chest CT showing areas of emphysema in the middle lobe a the lingula. LVR, lung volume reduction; CT, computed tomography; COPD, chronic obstructive pulmonary disease; GOLD, Global Initiative for Chronic Obstructive Lung Disease; DECT, dual-energy CT.

quantification is highly reproducible, when using identical scan parameters and reconstruction algorithms. However, it has not been shown that automated quantification of the extent and distribution of emphysema offers significant advantage in predicting if a patient has benefit when undergoing LVR over the evaluation by an experienced radiologist using a semiquantitative scoring approach (12).

Currently standardized quantitative methods and general guidelines for the assessment of quantitative CT are not available, although they are routinely used in the clinical practice.

\section{New techniques}

Novel techniques for the quantification of emphysema are based on DECT. This method can assess pulmonary perfusion by measuring iodine distribution maps or pulmonary ventilation by measuring the distribution of inhaled xenon gas (Figures 4,5) (38). The basis of this method is the acquisition of images at different X-ray 

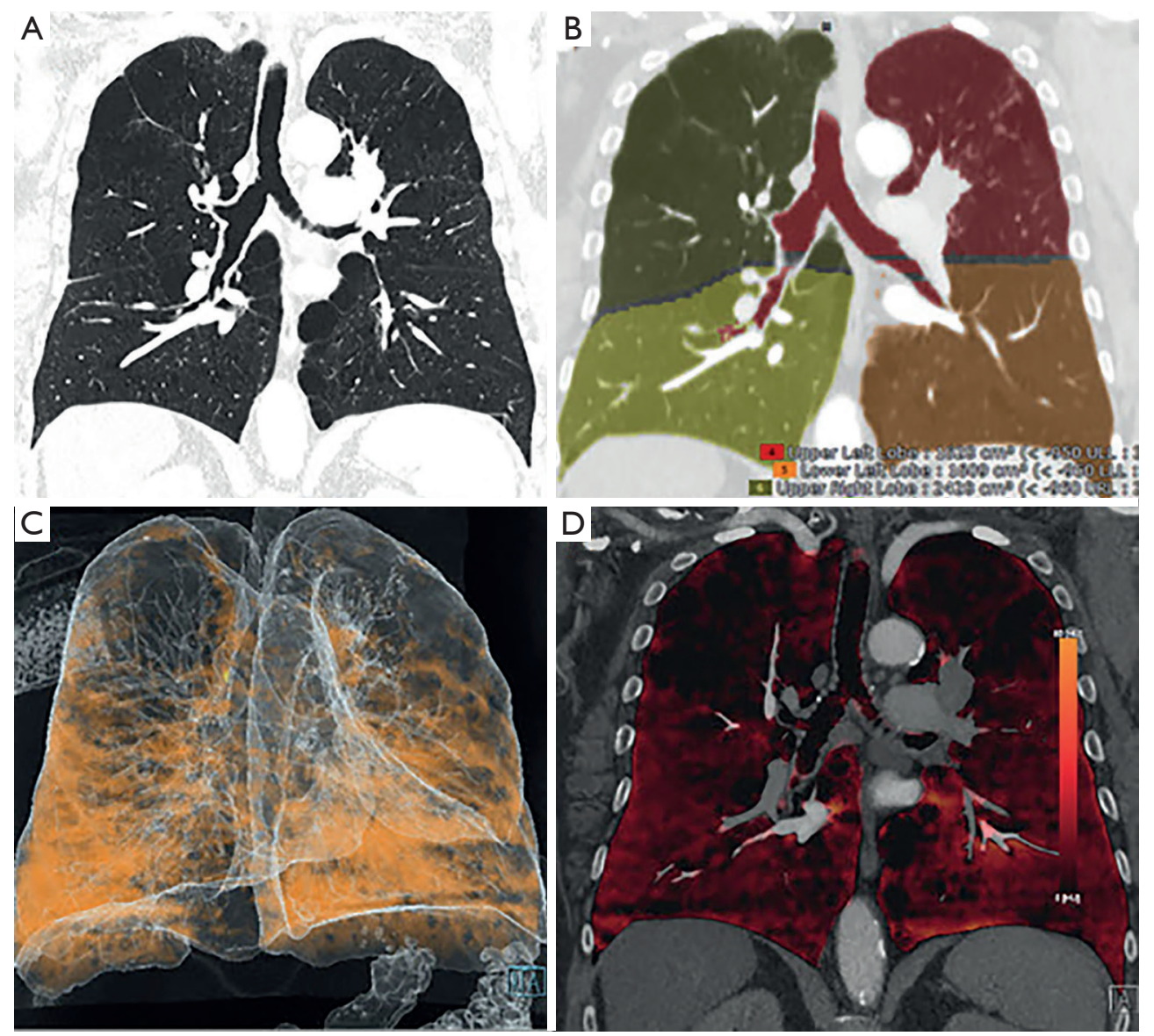

Figure 5 Pre-operative imaging before LVR. Preoperative evaluation of a 67-year-old male patient with COPD GOLD III: (A) coronal reconstruction of non-enhanced chest CT shows upper lobe predominant emphysema, correlating with areas of lower attenuation on (D) coronal reconstruction of DECT and (C) 3D reconstruction of the same DECT dataset. (B) It shows preoperative segmentation of the lungs in the different lobes. LVR, lung volume reduction; CT, computed tomography; COPD, chronic obstructive pulmonary disease; GOLD, Global Initiative for Chronic Obstructive Lung Disease; DECT, dual-energy CT.

energies and analysis of attenuation differences, allowing to create iodine maps of the lung parenchyma reflecting the perfusion, which can provide additional physiological information in patients with COPD beyond the pure morphological assessment with standard CT (39). This novel method in the diagnostic accuracy of standard CT leads to a one-stop-shop tool while maintaining its advantages over scintigraphy, but adding the structural information.

Xenon enhanced DECT (XE-DECT) studies demonstrated that lung zones enhanced by xenon strongly correlate with obstructive ventilation impairment (40). Similarly, ventilation defects seen on XE-DECT correlate significantly with airflow obstruction on LFEs $(22,41,42)$ and can be used for visualization of the distribution and localization of emphysema in COPD. A recent study by Lee et al. concluded that the parenchymal attenuation change between inspiration/expiration CTs and xenon dynamic change correlate significantly, and might be more robust as lung function parameters except FEV1 (22).

\section{Role of scintigraphy}

Another technique, which can be employed to evaluate patients with severe emphysema who are candidates for LVR is scintigraphy (Figure 6). Scintigraphy allows for visualizing ventilation and perfusion of the lungs and is therefore able to show upper-lobe predominance and 

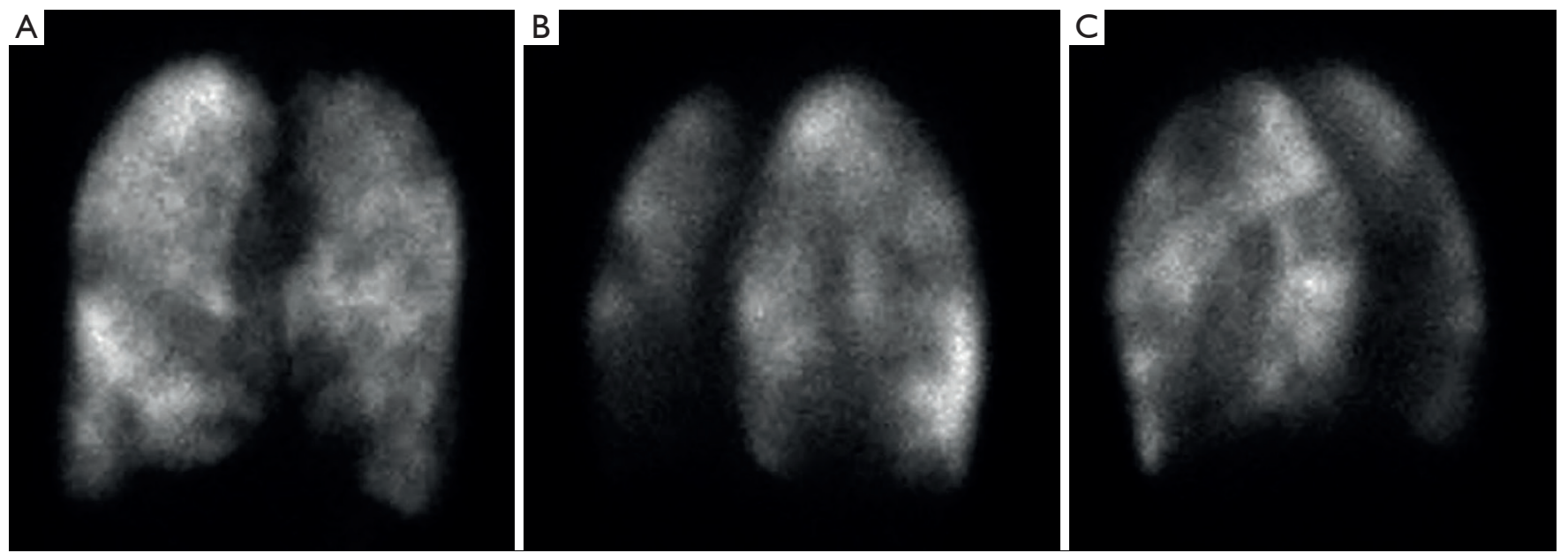

Figure 6 Example of lung scintigraphy. A 74-year-old male patient with COPD GOLD IV shows perfusion maps in (A) anteroposterior, (B) right oblique and (C) left oblique perfusion maps. Areas with reduced or no tracer uptake correspond to emphysematous areas of the lung. COPD, chronic obstructive pulmonary disease; GOLD, Global Initiative for Chronic Obstructive Lung Disease.

heterogeneity of emphysema. Lung scintigraphy is helpful to identify the most severely affected parts of the lungs in heterogeneous emphysema, but struggles in cases of homogeneous emphysema (43). Quantification is done by dividing the lung in three distinct areas (i.e., upper, middle and lower area) and calculating the percentage of uptake of tracers divided into ventilation and perfusion for each particular zone.

Several authors showed the high accordance between lung perfusion assessed by HRCT and on scintigraphy, questioning the additional information scintigraphy for lung evaluation making the method superfluous in LVR evaluation as mentioned above $(7,44)$.

\section{Role of MRI}

MRI of the lung is the latest imaging tool been introduced for the assessment of lung changes. Currently MR still plays only a minor role in clinical setting. MRI is mainly used as an alternative imaging modality in cases where radiation exposure plays a relevant role. However, in the last years due to new sequences the role of MR is changing and the method more and more achieved a central role in lung imaging. MRI is more than any other imaging method capable to combine morphologic and functional information $(45,46)$. Even though CT might be better in visualizing subtle changes in lung parenchyma, the strength of MRI is functional imaging: MRI is able to visualize ventilation and time resolved lung perfusion, it can show breathing dynamic and functional imaging of the diaphragm.

Lung morphology and function can also be evaluated using oxygen enhanced MRI or nobel gases: hyperpolarized noble gases or molecular oxygen can be used as an inhaled MRI contrast agent which is able to show regional ventilation and perfusion.

Similarly, with the administration of blood contrast media the signal of the lung during dynamic MRI can be postprocessed with the use of calculation of inflow over time in order to obtain quantitative parameters for pulmonary blood flow, blood volume and mean transit time $(47,48)$. These quantitative maps of lung perfusion obtained on MR are comparable to perfusion scintigraphy $(49,50)$. Dynamic contrast-enhanced MRI has also been propounded for the evaluation and monitoring of COPD disease severity (51).

Fourier decomposition lung MRI, a recently introduced technique for functional lung imaging has the ability to examine the patient without the use of contrast agents. It allows the simultaneous assessment of regional lung perfusion and ventilation-related information (52). Although the acquisition last only 30 seconds and the results are promising, the method is not very robust, resulting in too many artifacts (53).

Furthermore, MRI is a viable method for the assessment of lung volumes and respiratory mechanics: beside the upper-lobe predominance and heterogeneity, MRI is also able to visualize impaired respiratory motion of the chest wall and the diaphragm, which are additional factors in 
COPD. Preliminary results of Suga et al. (54) showed that with dynamic MRI it is possible to non-invasively visualize dynamic respiratory motions of the diaphragm and chest wall and to quantify post-operative improvement or worsening of patients undergoing LVR and is therefore potentially useful for monitoring the effects of LVR (55).

\section{Which patients should be operated?}

In a number of large case series, LVR proved to reduce dyspnoea and to improve lung function and wellbeing in patients with advanced pulmonary emphysema. The NETT trial confirmed these findings and demonstrated that selected patients live longer after surgery. Since patient selection is crucial to the success of the procedure, treatment should be performed at a specialised centre with a multidisciplinary team approach on emphysema treatment (12).

The goal of LVR is to remove emphysematous and hyper-inflated parts of the lung in order to restore respiratory mechanics, to lower air trapping and residual volume resulting in better ventilation and increased patients' wellbeing. While studies (12) show that patients with heterogeneous or upper lobe predominant emphysema profit from LVR, other meta-analyses showed that patients with homogeneous distribution of emphysema did not show benefit from surgery $(56,57)$.

Contraindications for LVR are severe bronchiectasis, large bullae, massive destruction of lung parenchyma and malignancy.

Therefore, it is important to assess preoperatively heterogeneity and distribution of emphysema in COPD and to rule out contraindications in order to find suitable candidates for LVR. CT is the standard of reference for assessment of emphysema distribution and for preoperative planning to predict outcome by simulating the resection. Quantitative CT may also be used to simulate the resection. Nowadays the different segmentation tools allow besides automated lung or lob segmentation also the semiautomated segmentation of areas. This enables to analyse the influence of resection zones by the thoracic surgeon on the changes of destructed lung volume.

Combining surgical planning on quantitative CT with parameters from lung function, dynamic MRI and perfusion from DECT will allow to predict the outcome and therewith a better selection of patients, but also allows to stretch the boarders may including patients that currently would not have been operated.

\section{Role of imaging for endobronchial LVR (valves, coils)}

Less invasive techniques than LVR are the endobronchial placement of valves and coils. The goal is to block regional inflation, while allowing exhalation in order to reduce air trapping and improve lung function (15). The CTevaluation of possible candidates undergoing endobronchial LVR contains (I) the assessment of the severity and anatomic distribution of emphysema (II) the identification of the target lobe and (III) the assessment of the completeness of interlobar fissures. Santos et al. (58) showed that emphysema distribution and fissure integrity were next to the lung function the most important factors to predict treatment success. Fissures anatomy must be addressed before performing LVR procedures of COPD as a marker for interlobar collateral ventilation as shown above: patients with severe emphysema and complete fissures depicted on CT had a better response to endobronchial valve placement $(2,58)$. Incomplete fissures constitute interlobar collaterals and reduce the positive effects of lobar isolation by EBV placement (15). These findings support the use of quantitative HRCT in choosing patients which will benefit the most from EBV therapy (15). Nowadays computerized methods to automatically allowing the quantification of fissural integrity are already in use (Figure 7) (36).

Endobronchial LVR utilizing one-way endobronchial valves is an accepted treatment for patients with in both heterogeneous upper and lower lobe predominant emphysema and without interlobar collateral ventilation.

Despite its beneficial, physiologic role in preventing atelectasis, collateral ventilation is limiting therapeutic success in Endobronchial LVR with valves in severe emphysema, whereas LVR coils and polymeric LVR are considered independent of collateral ventilation.

Therefore, for patients with collateral ventilation, who are not considered candidates for valve treatment, and for patients with homogeneous emphysema, the introduction of LVR coil treatment is a promising solution. The use of biochemical sealant is restricted to emphysema of upperlobe predominance.

After endobronchial LVR procedure, the indications for a chest radiography or chest CT include a check for pneumothorax, control of adequate placement of implanted devices, detection of device migration, evaluation of therapeutic success or any subsequent clinical encounters. 


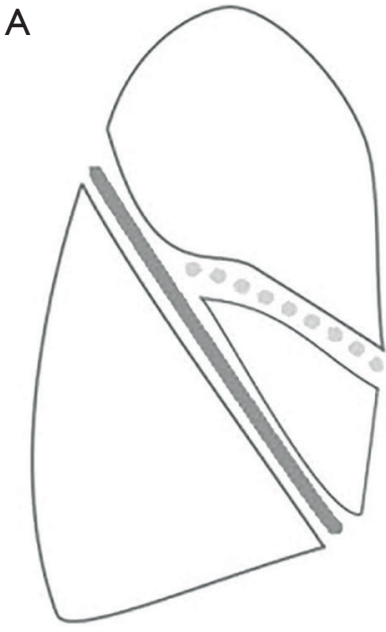

B

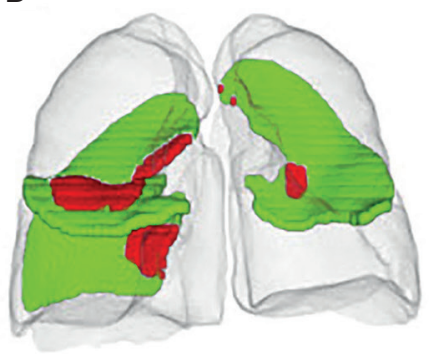

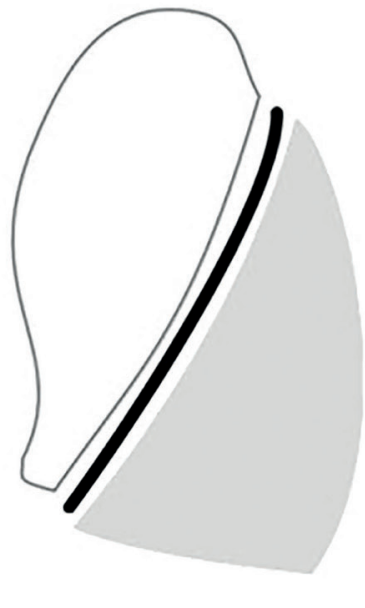

C

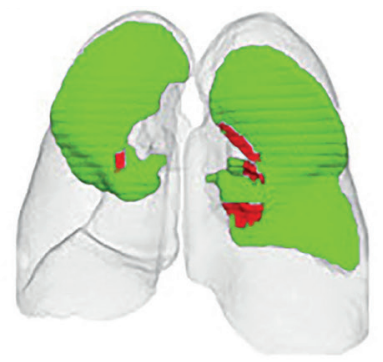

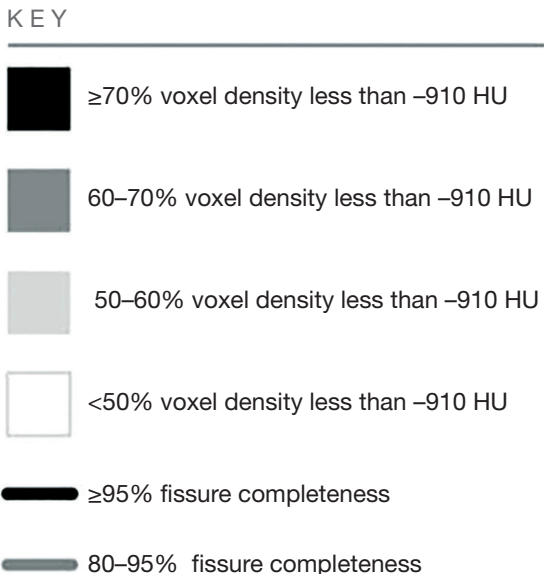

. $<80 \%$ fissure completeness

D

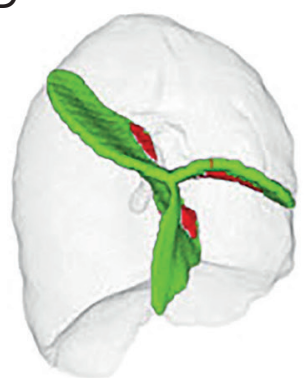

$\mathrm{E}$

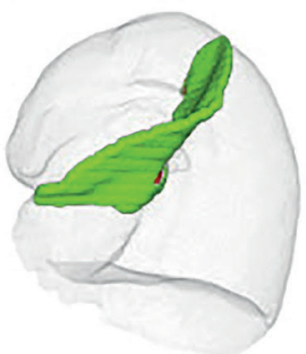

Figure 7 Pre-operative imaging before LVR. Figure shows quantitative fissure analysis performed with StratXTM Lung report software (Thirona LungQ, Veriosn 1.0, Pulmonx, USA). (A) This shows a summary of lung density as well as fissure analysis with $>95 \%$ fissure completeness in the left oblique fissure, between $80 \%$ and $95 \%$ fissure completeness in the right oblique fissure and less than $80 \%$ fissure completeness in the horizontal fissure. In (B) frontal view, (C) back view, (D) right lateral view and (E) left lateral view incomplete fissures are highlighted in red. LVR, lung volume reduction.

\section{Conclusions}

Besides clinical examination and LFT, imaging is one of the key factors for the success of surgical or minimal invasive therapy of severe lung emphysema. Especially CT with its increasing possibilities in assessing lung structure has brought additional insides into the pathology and supports thoracic surgeons and interventional pneumologists in selecting patients and optimising LVR procedures but also enables the development of new endovascular therapies. Emerging techniques as MR and DECT will further improve the individual outcome by supporting the choice of optimal therapy.

\section{Acknowledgements}

None.

\section{Footnote}

Conflicts of Interest: The authors have no conflicts of interest to declare.

\section{References}

1. Global Initiative for Chronic Obstructive Lung Disease (GOLD), 2017. Global Strategy for the Diagnosis, Management and Prevention of COPD. Available online: 
http://goldcopd.org/gold-2017-global-strategy-diagnosismanagement-prevention-copd/

2. Santos S, Peinado VI, Ramirez J, et al. Characterization of pulmonary vascular remodelling in smokers and patients with mild COPD. Eur Respir J 2002;19:632-8.

3. Wells JM, Washko GR, Han MK, et al. Pulmonary arterial enlargement and acute exacerbations of COPD. N Engl J Med 2012;367:913-21.

4. Mohamed Hoesein FA, de Jong PA, Lammers JW, et al. Contribution of CT quantified emphysema, air trapping and airway wall thickness on pulmonary function in male smokers with and without COPD. COPD 2014;11:503-9.

5. Xia Y, Guan Y, Fan L, et al. Dynamic contrast enhanced magnetic resonance perfusion imaging in high-risk smokers and smoking-related COPD: correlations with pulmonary function tests and quantitative computed tomography. COPD 2014;11:510-20.

6. Ingrisch M, Maxien D, Schwab F, et al. Assessment of pulmonary perfusion with breath-hold and freebreathing dynamic contrast-enhanced magnetic resonance imaging: quantification and reproducibility. Invest Radiol 2014;49:382-9.

7. Thurnheer R, Engel H, Weder W, et al. Role of lung perfusion scintigraphy in relation to chest computed tomography and pulmonary function in the evaluation of candidates for lung volume reduction surgery. Am J Respir Crit Care Med 1999;159:301-10.

8. Roach DJ, Cremillieux Y, Serai SD, et al. Morphological and quantitative evaluation of emphysema in chronic obstructive pulmonary disease patients: A comparative study of MRI with CT. J Magn Reson Imaging 2016;44:1656-63.

9. Jang YM, Oh YM, Seo JB, et al. Quantitatively assessed dynamic contrast-enhanced magnetic resonance imaging in patients with chronic obstructive pulmonary disease: correlation of perfusion parameters with pulmonary function test and quantitative computed tomography. Invest Radiol 2008;43:403-10.

10. Hoffman EA, Lynch DA, Barr RG, et al. Pulmonary CT and MRI phenotypes that help explain chronic pulmonary obstruction disease pathophysiology and outcomes. J Magn Reson Imaging 2016;43:544-57.

11. Cooper JD, Patterson GA, Sundaresan RS, et al. Results of 150 consecutive bilateral lung volume reduction procedures in patients with severe emphysema. J Thorac Cardiovasc Surg 1996;112:1319-29; discussion 1329-30.

12. Fishman A, Martinez F, Naunheim K, et al. A randomized trial comparing lung-volume-reduction surgery with medical therapy for severe emphysema. N Engl J Med 2003;348:2059-73.

13. Herth FJ, Noppen M, Valipour A, et al. Efficacy predictors of lung volume reduction with Zephyr valves in a European cohort. Eur Respir J 2012;39:1334-42.

14. Hunsaker AR, Ingenito EP, Reilly JJ, et al. Lung volume reduction surgery for emphysema: correlation of CT and V/Q imaging with physiologic mechanisms of improvement in lung function. Radiology 2002;222:491-8.

15. Sciurba FC, Ernst A, Herth FJ, et al. A randomized study of endobronchial valves for advanced emphysema. N Engl J Med 2010;363:1233-44.

16. Stolk J, Versteegh MI, Montenij LJ, et al. Densitometry for assessment of effect of lung volume reduction surgery for emphysema. Eur Respir J 2007;29:1138-43.

17. Ingenito EP, Loring SH, Moy ML, et al. Physiological characterization of variability in response to lung volume reduction surgery. J Appl Physiol (1985) 2003;94:20-30.

18. Rogers RM, Coxson HO, Sciurba FC, et al. Preoperative severity of emphysema predictive of improvement after lung volume reduction surgery: use of CT morphometry. Chest 2000;118:1240-7.

19. Davey C, Zoumot Z, Jordan S, et al. Bronchoscopic lung volume reduction with endobronchial valves for patients with heterogeneous emphysema and intact interlobar fissures (the BeLieVeR-HIFi study): a randomised controlled trial. Lancet 2015;386:1066-73.

20. Hetzel J, Boeckeler M, Horger M, et al. A new functional method to choose the target lobe for lung volume reduction in emphysema - comparison with the conventional densitometric method. Int J Chron Obstruct Pulmon Dis 2017;12:2621-8.

21. Flaherty KR, Kazerooni EA, Curtis JL, et al. Shortterm and long-term outcomes after bilateral lung volume reduction surgery: prediction by quantitative CT. Chest 2001;119:1337-46.

22. Lee SM, Seo JB, Hwang HJ, et al. Assessment of regional emphysema, air-trapping and Xenon-ventilation using dual-energy computed tomography in chronic obstructive pulmonary disease patients. Eur Radiol 2017;27:2818-27.

23. Gierada DS, Hakimian S, Slone RM, et al. MR analysis of lung volume and thoracic dimensions in patients with emphysema before and after lung volume reduction surgery. AJR Am J Roentgenol 1998;170:707-14.

24. Ohno Y, Nishio M, Koyama H, et al. Oxygen-enhanced MRI, thin-section MDCT, and perfusion SPECT/CT: comparison of clinical implications to patient care for lung volume reduction surgery. AJR Am J Roentgenol 
2012;199:794-802 .

25. Washko GR, Hoffman E, Reilly JJ. Radiographic Evaluation of the Potential Lung Volume Reduction Surgery Candidate. Proceedings of the American Thoracic Society 2008;5:421-6.

26. Thurlbeck WM, Simon G. Radiographic appearance of the chest in emphysema. AJR Am J Roentgenol 1978;130:429-40.

27. Muller N, Coxson H. Chronic obstructive pulmonary disease. 4: imaging the lungs in patients with chronic obstructive pulmonary disease. Thorax 2002;57:982-5.

28. Bankier AA, De Maertelaer V, Keyzer C, et al. Pulmonary emphysema: subjective visual grading versus objective quantification with macroscopic morphometry and thinsection CT densitometry. Radiology 1999;211:851-8.

29. Hersh CP, Washko GR, Jacobson FL, et al. Interobserver variability in the determination of upper lobe-predominant emphysema. Chest 2007;131:424-31.

30. Coxson HO, Rogers RM, Whittall KP, et al. A quantification of the lung surface area in emphysema using computed tomography. Am J Respir Crit Care Med 1999;159:851-6.

31. Gevenois PA, de Maertelaer V, De Vuyst P, et al. Comparison of computed density and macroscopic morphometry in pulmonary emphysema. Am J Respir Crit Care Med 1995;152:653-7.

32. Gevenois PA, Koob MC, Jacobovitz D, et al. Whole lung sections for computed tomographic-pathologic correlations. Modified Gough-Wentworth technique. Invest Radiol 1993;28:242-6.

33. Washko GR, Martinez FJ, Hoffman EA, et al. Physiological and computed tomographic predictors of outcome from lung volume reduction surgery. Am J Respir Crit Care Med 2010;181:494-500.

34. Madani A, Van Muylem A, de Maertelaer V, et al. Pulmonary emphysema: size distribution of emphysematous spaces on multidetector CT images-comparison with macroscopic and microscopic morphometry. Radiology 2008;248:1036-41.

35. Mantri S, Macaraeg C, Shetty S, et al. Technical advances: measurement of collateral flow in the lung with a dedicated endobronchial catheter system. J Bronchology Interv Pulmonol 2009;16:141-4.

36. Schuhmann M, Raffy P, Yin Y, et al. Computed tomography predictors of response to endobronchial valve lung reduction treatment. Comparison with Chartis. Am J Respir Crit Care Med 2015;191:767-74.

37. Koenigkam-Santos M, Puderbach M, Gompelmann D, et al. Incomplete fissures in severe emphysematous patients evaluated with MDCT: incidence and interobserver agreement among radiologists and pneumologists. Eur J Radiol 2012;81:4161-6.

38. Kauczor HU, Wielputz MO, Owsijewitsch M, et al. Computed tomographic imaging of the airways in COPD and asthma. J Thorac Imaging 2011;26:290-300.

39. Hagspiel KD, Flors L, Housseini AM, et al. Pulmonary blood volume imaging with dual-energy computed tomography: spectrum of findings. Clin Radiol 2012;67:69-77.

40. Sugino K, Kobayashi M, Nakamura Y, et al. XenonEnhanced Dual-Energy CT Imaging in Combined Pulmonary Fibrosis and Emphysema. PLoS One 2017;12:e0170289.

41. Chae EJ, Seo JB, Lee J, et al. Xenon ventilation imaging using dual-energy computed tomography in asthmatics: initial experience. Invest Radiol 2010;45:354-61.

42. Goo HW, Yang DH, Hong SJ, et al. Xenon ventilation CT using dual-source and dual-energy technique in children with bronchiolitis obliterans: correlation of xenon and CT density values with pulmonary function test results. Pediatr Radiol 2010;40:1490-7.

43. Wang SC, Fischer KC, Slone RM, et al. Perfusion scintigraphy in the evaluation for lung volume reduction surgery: correlation with clinical outcome. Radiology 1997;205:243-8.

44. Cleverley JR, Desai SR, Wells AU, et al. Evaluation of patients undergoing lung volume reduction surgery: ancillary information available from computed tomography. Clin Radiol 2000;5 5:45-50.

45. Ley-Zaporozhan J, Puderbach M, Kauczor HU. MR for the evaluation of obstructive pulmonary disease. Magn Reson Imaging Clin N Am 2008;16:291-308, ix.

46. Puderbach M, Eichinger M, Haeselbarth J, et al. Assessment of morphological MRI for pulmonary changes in cystic fibrosis (CF) patients: comparison to thin-section CT and chest x-ray. Invest Radiol 2007;42:715-25.

47. Fink C, Puderbach M, Bock M, et al. Regional lung perfusion: assessment with partially parallel threedimensional MR imaging. Radiology 2004;231:175-84.

48. Ohno Y, Hatabu H, Murase K, et al. Quantitative assessment of regional pulmonary perfusion in the entire lung using three-dimensional ultrafast dynamic contrastenhanced magnetic resonance imaging: Preliminary experience in 40 subjects. J Magn Reson Imaging 2004;20:353-65.

49. Yilmaz E, Akkoclu A, Degirmenci B, et al. Accuracy and 
feasibility of dynamic contrast-enhanced 3D MR imaging in the assessment of lung perfusion: comparison with Tc-99 MAA perfusion scintigraphy. Clin Radiol 2005;60:905-13.

50. Molinari F, Fink C, Risse F, et al. Assessment of differential pulmonary blood flow using perfusion magnetic resonance imaging: comparison with radionuclide perfusion scintigraphy. Invest Radiol 2006;41:624-30.

51. Sergiacomi G, Sodani G, Fabiano S, et al. MRI lung perfusion 2D dynamic breath-hold technique in patients with severe emphysema. In Vivo 2003;17:319-24.

52. Wujcicki A, Corteville D, Materka A, et al. Perfusion and ventilation filters for Fourier-decomposition MR lung imaging. Z Med Phys 2015;25:66-76.

53. Kjorstad A, Corteville DM, Henzler T, et al. Non-invasive quantitative pulmonary V/Q imaging using Fourier decomposition MRI at 1.5T. Z Med Phys 2015;25:326-32.

54. Suga K, Tsukuda T, Awaya H, et al. Impaired respiratory mechanics in pulmonary emphysema: evaluation with

Cite this article as: Martini K, Frauenfelder T. Emphysema and lung volume reduction: the role of radiology. J Thorac Dis 2018;10(Suppl 23):S2719-S2731. doi: 10.21037/jtd.2018.05.117 dynamic breathing MRI. J Magn Reson Imaging 1999; 10:510-20.

55. Sverzellati N, Molinari F, Pirronti T, et al. New insights on COPD imaging via CT and MRI. International Journal of Chronic Obstructive Pulmonary Disease 2007;2:301-12.

56. Slone RM, Pilgram TK, Gierada DS, et al. Lung volume reduction surgery: comparison of preoperative radiologic features and clinical outcome. Radiology 1997;204:685-93.

57. Berger RL, Wood KA, Cabral HJ, et al. Lung volume reduction surgery: a meta-analysis of randomized clinical trials. Treat Respir Med 2005;4:201-9.

58. Koenigkam-Santos M, de Paula WD, Owsijewitsch M, et al. Incomplete pulmonary fissures evaluated by volumetric thin-section CT: Semi-quantitative evaluation for small fissure gaps identification, description of prevalence and severity of fissural defects. European Journal of Radiology 2013;82:2365-70. 